\title{
Síndrome de Chilaiditi Associada a Volvo de Cólon Sigmóide - Relato de Caso
}

\author{
Chilaiditi’s Syndrome with Sigmoid Colon Volvulus - Case Report
}

\author{
MARCELO WILSON ROCHAALMEIDA ${ }^{1}$; BRUNO HELLWIG²; RICARDO LANZETTAHAACK ${ }^{3}$; \\ ANDRÉRODRIGUESDASILVA ${ }^{4}$
}

\begin{abstract}
${ }^{1}$ Médico-Residente em Cirurgia Geral do Hospital Universitário São Francisco de Paula (HUSFP). ${ }^{2}$ Títular da Sociedade Brasileira de Coloproctologia, Titular do Colégio Brasileiro de Cirurgiões e professor adjunto da Clínica Cirúrgica da Escola de Medicina da Universidade Católica de Pelotas (UCPel). ${ }^{3}$ Cirurgião Oncológico do Hospital Escola da Universidade Federal de Pelotas e Cirurgião do Pronto Socorro de Pelotas. ${ }^{4}$ Acadêmico da Escola de Medicina da Universidade Católica de Pelotas - RS - Brasil.
\end{abstract}

ALMEIDA MWR; HELLWIG B; HAACK RL; SILVAAR. Síndrome de Chilaiditi Associada a Volvo de Cólon Sigmóide - Relato de Caso. Rev bras Coloproct, 2006;26(4): 449-453.

RESUMO: Introdução: Este estudo tem como objetivo relatar um caso de Síndrome de Chilaiditi associada a volvo de cólon sigmóide. Relato de caso: Paciente masculino, branco, 51 anos, admitido no Pronto-Socorro de Pelotas queixando parada da eliminação de gases e fezes, dor abdominal difusa com distensão e inapetência, aceitando somente líquido. Tem diagnóstico de retardo mental e constipação intestinal crônica. Foi realizado Rx de abdome agudo, sendo evidenciada distensão difusa de cólon, e sinais sugestivos de volvo de sigmóide com imagem de cólon transverso entre o fígado e o diafragma. $O$ paciente foi submetido a laparotomia exploradora, sendo constatado volvo de sigmóide, megacólon difuso e interposição do cólon transverso entre o fígado e o diafragma. Procedeu-se a colectomia subtotal, com colostomia terminal em cólon ascendente e fechamento do reto remanescente, recebendo alta no $9^{\circ}$ dia. Discussão: A interposição do cólon entre o fígado e a cúpula diafragmática (síndrome de Chilaiditi), associada a volvo de cólon sigmóide, constitui causa rara de abdome agudo obstrutivo, embora o volvo de sigmóide seja uma das principais causas de obstrução intestinal mecânica no Brasil. Geralmente o tratamento é clínico, porém se associado a complicações o tratamento é cirúrgico.

Descritores: Síndrome de Chilaiditi; volvo; cólon; sinal de Chilaiditi.

\section{INTRODUÇÃO}

A interposição temporária ou permanente do cólon, intestino delgado (raro) ou estômago (raríssimo) no espaço hepatodiafragmático é conhecida como sinal de Chilaiditi, sendo geralmente assintomática ${ }^{(1)}$. É evidenciada normalmente em um exame radiológico de abdome de rotina. A sua incidência é de $0,025 \%$ nos exames de raios-X em qualquer faixa etária, aumentando levemente nos maiores de 60 anos, sendo mais comum em homens do que em mulheres na relação de $4: 1^{(1-4)}$.
Demetrius Chilaiditi em 1910 relatou 3 (três) casos de pacientes assintomáticos com interposição hepatodiafragmática do cólon ${ }^{(1-7)}$. A associação do sinal de Chilaiditi com sintomas, como dor abdominal, náuseas, dor retroesternal, sintomas respiratórios, vômitos, distensão abdominal, obstrução ou suboclusão intestinal caracteriza a Síndrome de Chilaiditi ${ }^{(1-7)}$.

A associação de volvo de cólon com a Síndrome de Chilaiditi é rara, tendo sido publicados 7 (sete) casos na literatura internacional (língua inglesa), 6 (seis) adultos em sua maioria homens e 1 (um) numa criança de $12 \operatorname{anos}^{(1)}$.

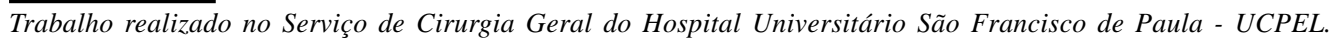

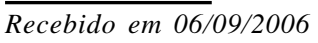

Aceito para publicação em 22/11/2006 
O presente relato apresenta um novo caso da síndrome de Chilaiditi associada a volvo de cólon sigmóide, tratado cirurgicamente no Serviço de Cirurgia Geral do HUSFP em 2006.

\section{RELATO DO CASO}

Paciente masculino, branco, solteiro, 51 anos, natural e residente em Pelotas, RS. Procurou o Pronto-Socorro de Pelotas (PSP) com queixa de constipação, inapetência gradual e progressiva, aceitando somente dieta liquida há 5 (cinco) dias. Relatava parada de eliminação de gases, dor abdominal difusa, vômitos e aumento do volume abdominal nas últimas $24 \mathrm{hs}$. Ao exame físico, apresentava-se desidratado, com fácies de sofrimento, distensão abdominal importante, timpânico, ruídos hidroaéreos ausentes, dor à palpação superficial e profunda com sinais de irritação peritonial difusamente. Toque retal sem fezes na ampola retal e/ ou alterações ao alcance do dedo.

Familiar referia que o paciente apresenta-se, desde a adolescência, com quadro de constipação intestinal, com fezes de consistência endurecida, sem muco, pús ou sangue. Relatava que o paciente era medicado, por conta de familiares, com laxativos na freqüência de 1 a 2 vezes por semana. Há aproximadamente 2 anos, iniciou, conforme orientação medica, dieta rica em fibras e cereais e há 8 meses parou o uso de laxativos.

Apresenta diagnóstico de retardo mental feito aos 8 anos de idade e desde então faz uso de haloperidol $5 \mathrm{mg} /$ dia. Desde a infância tem dificuldade para comunicar-se, deambular sozinho, sem controle esfincteriano. Nega cirurgia e internação prévia. Referia também que a mãe era hipertensa, nefropata e constipada crônica, tendo sido submetida a cirurgia abdominal com realização de colostomia, não sabendo o porquê. Pai faleceu de infarto agudo do miocárdio fulminante

Foi solicitado hemograma que evidenciou hemácias 5.09, hemoglobina 13.4, hematócrito 40.3\%, VCM 79, leucócitos 13.900, bastões 1\%, segmentados $85 \%$, eosinófilos $1 \%$, monócitos $4 \%$, linfócitos $9 \%$. Foi realizado $\mathrm{Rx}$ de abdome agudo, sendo evidenciada distensão de cólon, sugestiva de volvo de sigmóide, com imagem de cólon transverso entre o fígado e o diafragma. (Figuras 1, 2, 3).

O paciente foi submetido a laparotomia exploradora com incisão mediana, sendo constatado megacólon com volvo de sigmóide, e interposição do

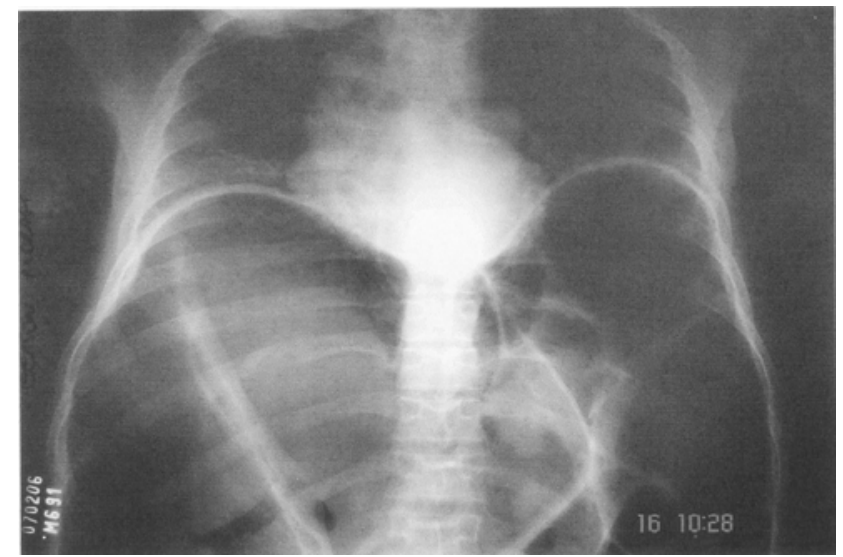

Figura 1 - Rx de abdome agudo evidenciando cólon transverso entre o fígado e o diafragma.

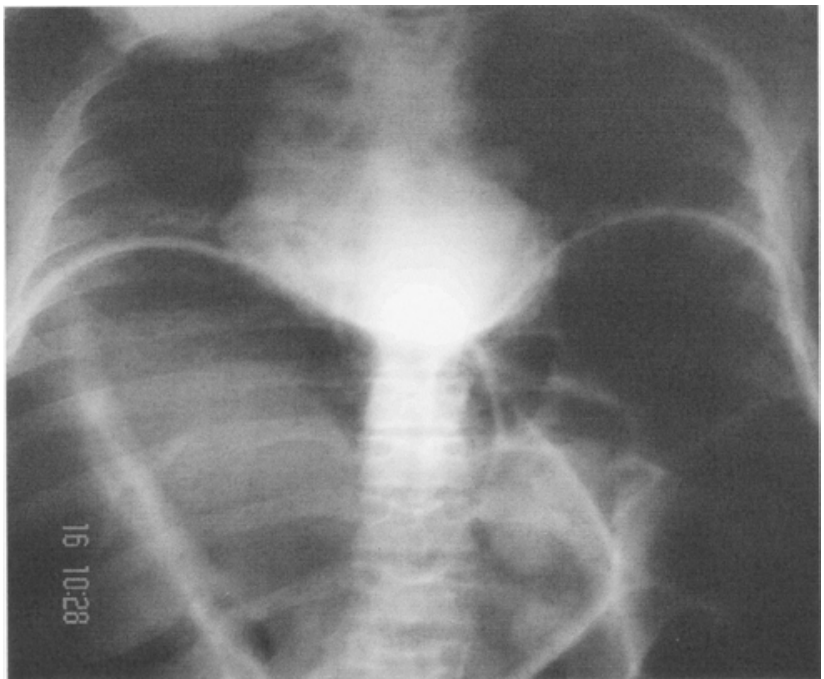

Figura 2 - Rx de abdome agudo evidenciando cólon transverso entre o fígado e o diafragma.

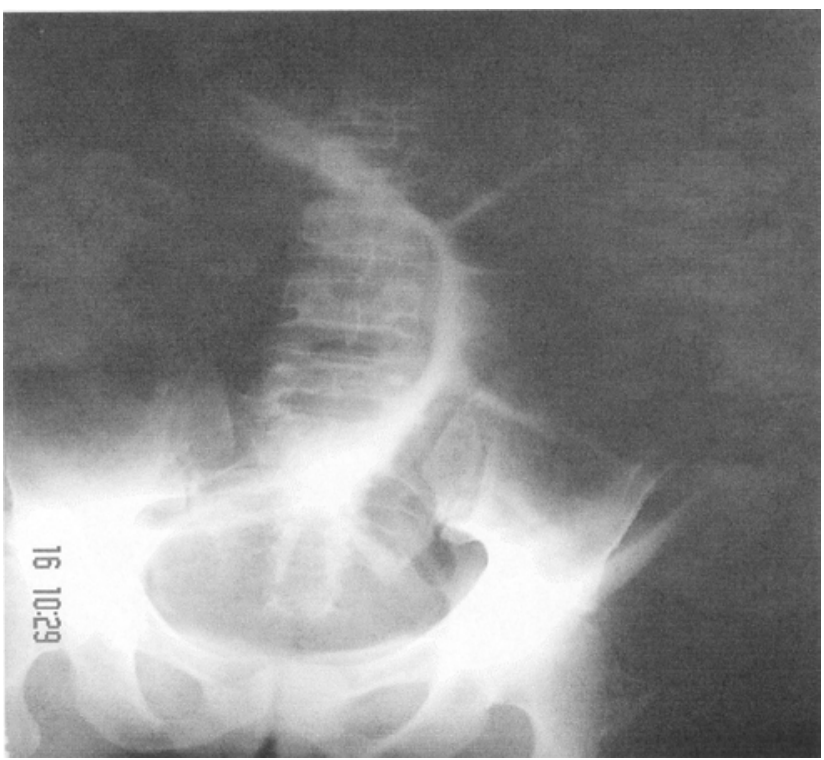

Figura 3 - Rx de abdome agudo evidenciando volvo de sigmóide. 
cólon transverso entre o fígado e o diafragma. (Figuras 4 e 5). Procedeu-se a colectomia subtotal, com colostomia terminal em cólon ascendente e fechamento do coto retal remanescente. (Figura 6). O paciente evolui favoravelmente recebendo alta no $9^{\circ}$ dia de pósoperatório.

A histopatologia evidenciou cólon com acentuada atrofia mural e áreas de infarto hemorrágico. Células ganglionares mioentéricas presentes e inalteradas. Linfonodos com intensa congestão e linfangectasias.

Paciente no sexto mês de pós-operatório sem intercorrências. Foi lhe sugerido o restabelecimento do trânsito intestinal e ele ainda não concordou com tal conduta que, obviamente, é a adequada.

\section{DISCUSSÃO}

Na literatura médica, são descritos três tipos de interposição hepatodiafragmática: interposição do cólon transverso e/ou intestino delgado no espaço subfrênico anterior direito, interposição do cólon ou estômago no espaço extraperitoneal direito e interposição do cólon transverso no espaço sub-frênico posterior direito. $^{(3)}$

São descritos muitos fatores predisponentes, os mais freqüentes relacionados com possíveis alterações anatômicas entre fígado, cólon e diafragma, como: diminuição do tamanho do fígado, defeitos congênitos dos ligamentos hepáticos, má-rotação ou mobilidade anormal do intestino, alongamento do cólon ou estreitamento da inserção no mesentério, alteração do nervo frênico, eventração diafragmática e aumento do diâmetro torácico. ${ }^{(4)}$ Outros fatores também relatados: constipação crônica, cirurgia abdominal prévia, obesidade e aerofagia. ${ }^{(5)}$

Geralmente assintomática, esta circunstância é relatada como um achado ocasional em exame radiológico, sendo denominada de Sinal de Chilaiditi, mas pode ser acompanhada de dor abdominal, náuseas, vômitos, anorexia, constipação e menos geralmente associada a complicações severas, como obstrução intestinal aguda, sendo denominada de Síndrome de Chilaiditi. (2)

Em adultos, a síndrome costuma ser associada a cirrose, ascite ${ }^{(6)}$, enfisema, hipotireoidismo com constipação crônica, hipertensão arterial, coronariopatias, apendicite, ectopia renal, síndrome de Cushing ${ }^{(9)}$, esquizofrenia, retardo mental e uso de drogas antipsicóticas. $^{(7)}$

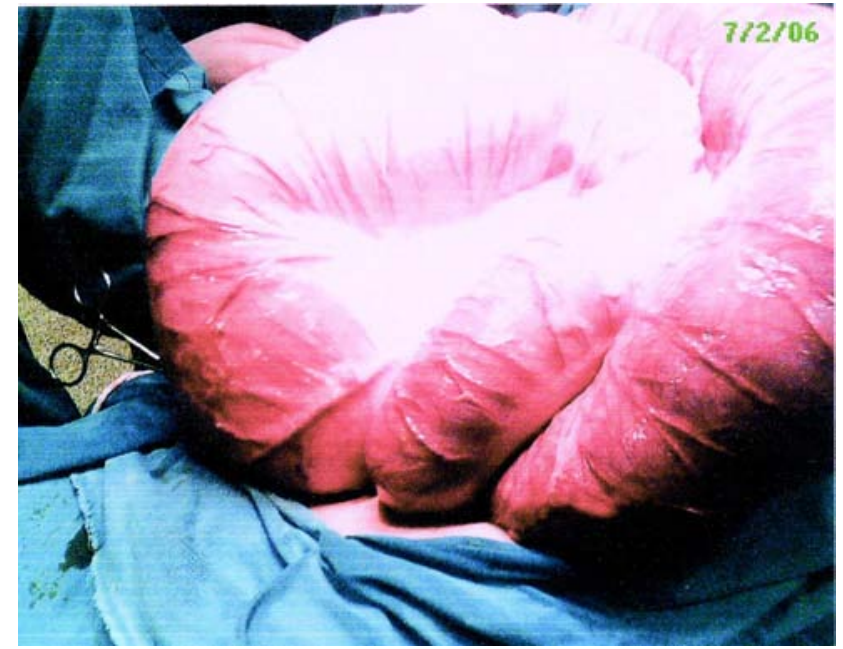

Figura 4-Megacólon com volvo de sigmóide.

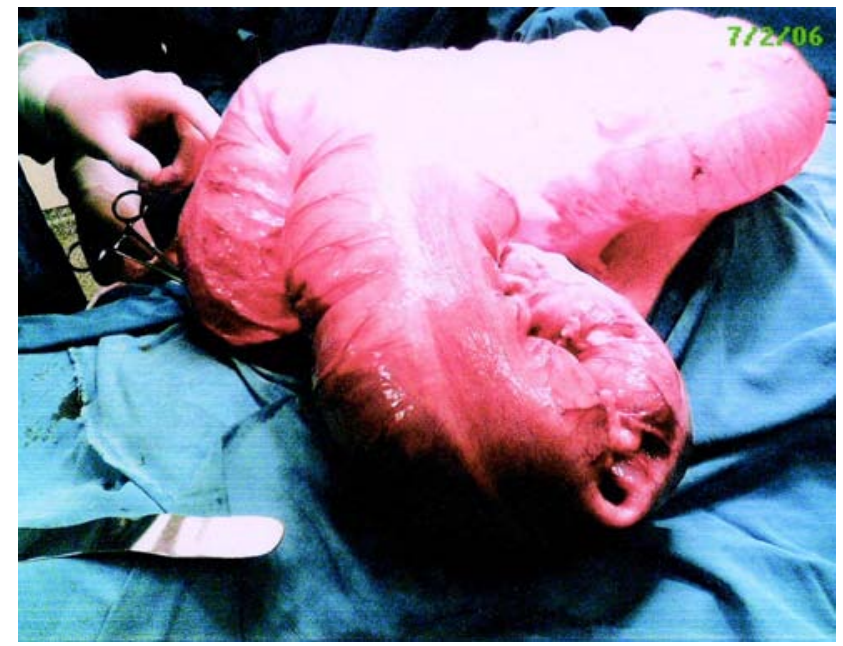

Figura 5-Megacólon com volvo de sigmóide.

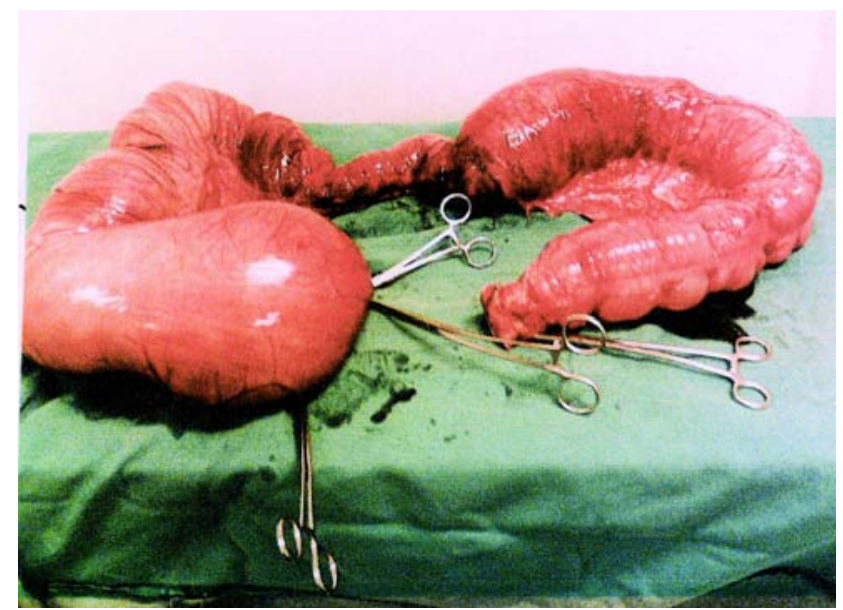

Figura 6-Megacólon. 
Nas crianças, parece existir relação com presença de alterações anatômicas congênitas. ${ }^{(4)} \mathrm{A}$ incidência dessa patologia varia de $0,025 \%$ a $0,28 \%$ na população, ${ }^{(8)}$ sendo 4 vezes mais freqüente em homens e acima de 65 anos. ${ }^{(2)}$

Em nosso caso, o paciente apresentava-se com retardo mental, uso crônico de medicação antipsicótica, constipação intestinal crônica e alongamento do cólon.

O volvo de cólon é uma conhecida causa de obstrução intestinal; aproximadamente $3 \%$ $5 \%$ de todos os casos de obstrução intestinal são produzidos por volvo de cólon e de todos os segmentos o cólon sigmóide está envolvido em 40 a $60 \%$. $^{(10)}$

O volvo de sigmóide ocorre em pacientes com cólon sigmóide alongado e redundante e com estreitamento em nível de mesentério. Sua elevada prevalência em pacientes internados em instituições psiquiátricas é um fator a ser considerado, devido à constipação crônica e medicações que normalmente são usadas nesses pacientes. No Brasil, a doença de Chagas com megacólon é uma causa importante de volvo de cólon. ${ }^{(11)}$

Atualmente a descompressão endoscópica é o procedimento de escolha para tratamento do volvo de sigmóide em paciente estável e sem sinais de isquemia ou perfuração do intestino, ${ }^{(12,13)}$ e pode resolver o qua- dro agudo em 70 a $90 \%$ dos casos, principalmente devido ao diagnóstico e tratamento precoce. Falhando a descompressão endoscópica ou havendo sinais de isquemia intestinal, a ressecção do segmento comprometido se impõe (procedimento de Hartman para urgência e não preparo colônico é a técnica cirúrgica mais utilizada). ${ }^{(14)}$

Embora a síndrome de Chilaiditi possa ser tratada de forma conservadora, com repouso no leito, descompressão nasogástrica, dieta líquida e enemas, o procedimento cirúrgico deve ser considerado, quando os sintomas não respondem à terapia conservadora ou há sinais de complicação. ${ }^{(15,16)}$

\section{CONCLUSÃO}

A interposição do cólon entre o fígado e a cúpula diafragmática (síndrome de Chilaiditi), associada a volvo de cólon sigmóide, constitui causa rara de abdome agudo obstrutivo, embora o volvo de sigmóide seja uma das principais causas de obstrução intestinal mecânica no Brasil. A maioria dos pacientes é constipada crônica. A síndrome está associada a cirrose, ascite, enfisema, obesidade, hipertensão arterial, hipotireoidismo, esquizofrenia, apendicite, rim ectópico, síndrome de Cushing e retardo mental. Geralmente o tratamento é clínico, porém se aparecem complicações o tratamento é cirúrgico.

\footnotetext{
ABSTRACT: Introduction: This study aims at reporting a case of Chilaiditi Syndrome associated to sigmoid colon volvulus. Case report: Male patient, white, 51 years old, arrives at an emergency hospital in Pelotas with complaints of stoping the elimination of gases, diffuse abdominal pain, increase of abdominal volume, gradual and progressive inappetence, accepting only liquid. He is diagnosed with mental retardation and a situation of intestinal constipation. An acute abdomen Rx highlighted a diffuse colon distension, suggestive of sigmoid volvulus with tranversum colon image between the liver and the diaphragm. The patient was submitted to exploratory laparotomy being highlighted sigmoid volvulus, diffuse megacolon and interposition of the transverse colon between the liver and the diaphragm. Subtotal colectomy with terminal ascending colon colostomy was proceeded. The patient evolved satisfactorily being discharged on the $9^{\text {th }}$ day. Discussion: The interposition of the colon between the liver and the diaphragmatic cupula (Chilaiditi syndrome) associated to sigmoid colon volvulus constitutes a rare cause of obstructive acute abdomen, though the sigmoid volvulus is one of the main causes of mechanic intestinal obstruction in Brazil. Usually a clinical treatment is done, although when associated to complications the treatment is surgical.
}

Key words: Chilaiditi syndrome; volvulus; colon; Chilaiditi sign.

\section{REFERÊNCIAS}

1. Chilaiditi D. Zur Frage der Hepatostose und Ptose im allgemeinem im Anschuss na Fälle von temporärer, partieller. Lebervelagung. Fortschr Geb Röntgenstr Nuklearmed Erganzungsband 1910; 16: 173-208.
2. Flores N; Ingar C; Sánchez J; Fernández J; Lazarte C; Málaga J; et al, Síndrome de Chilaiditi complicada com Vólvulo de Colon Transverso. Rev Gastroenterologia 2005; 25:279-284.

3. Van Everdingen KJ; Feldberg MA. Diagnostic image Chilaiditi syndrome. Ned Tijdschr Geneeskd 2001; 145 (42): 2026. 
4. Katagiri Y; Onitsuka A; Miyauchi T; Mimoto H; Hirose M; Shimazaki M; et al.. A case of hypogenisis of the median segment of the liver complicated with Chilaiditi syndrome. Nippon S G Zasshi 2001; 98 (1): 48-52.

5. Matthews J, Beck GW, Bowley DM. Chilaiditi syndrome and recurrent colonic volvulus. R Nav Med Serv 2001; 87 (2):111-2.

6. Bhattacharya PC, Bhattacharya AK, Dutta S, Mahanta N, Talukdar R. Chilaiditi syndrome with ascites. J Assoc Physicians India 2002;50(6):860-1.

7. Miyaoka T; Seno H; Itoga M; Ohshiro R; Inagaki T; Horiguchi $\mathrm{J}$; et al. Chilaiditi's syndrome associated to schizophrenia: 3 case reports. J Clin Psychiatry 2001; 62:58-29.

8. Orangio GR, Fazio VW, Winkelman E, McGonagle BA. The Chilaiditi syndrome and associated volvulus of the transverse colon. An indication for surgical therapy. Dis Colon Rectum 1986; 29:653-656.

9. Pranava V, Rathod NR. Chilaiditi syndrome with hipertension. J Assoc Physicians India 2001; 49 (5):589.

10. Frizelle EA, Wolff BG. Colonic volvulus. Adv Surg 1996; 29:131-9.
11. Hiltunen KM, Syrjä H, Matikainen M: Colonic volvulus. Diagnosis and results of treatment in 82 patients. Eur J Surg 1992; 158:607-611

12. Méndez AL, Castillo AC, Martinez AG. Obstrucción intestinal secundaria a vólvulo del ángulo esplénico del colon. Rev Esp Enf Dig 1998; 90 (2): 128-9.

13. Chiulli RA, Swantkowski TM. Sigmoid volvulus treated with endoscopic sigmoidopexy. Gast Endoscopy 1993; 39:194-6.

14. Friedman JD, Odland MD, Bobrick Mp. Experience with colonic volvulus. Dis Colon \& Rectum 1989; 32:607-611.

15. Altomare DF; Rinaldi M; Petrolino M; Sallustio PL; Guglielmi A; Pannarale OC, Chilaiditi's syndrome. Successful surgical correction by colopexy. Tech Coloproctol 2001; 5:173-175.

16. White JJ, Chavez EP, Souza J. Internal hernia of the transverse colon - Chilaiditi syndrome in a child. J Pediatr Surg 2002; 37(5): 802-4.

\section{Endereço para correspondência:}

\section{MARCELO WILSON ROCHA ALMEIDA}

Rua Santos Dumont, 548 - apto 301 - Ed. Camila. 96.020-380 - Pelotas, RS, Brasil

Tel.: (53) 3225-7151.

E-mail: mwralmeida@brturbo.com.br 